\title{
Chronic Non-Communicable Diseases and Health Technologies
}

\author{
Maria Lúcia Zanetti
}

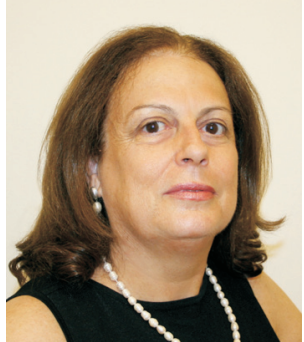

The increasing demand of people with chronic non-communicable diseases (CNCDs) currently poses a challenge for the health system in many countries. This challenge is related to the scarcity of health resources and the need to produce knowledge that can provide tools to permit an effective approach of that population and their risk factors, taking into account cost-effectiveness. It is recognized that health promotion actions increase health expenditures but, on the other hand, bring economic benefits to the countries in the long term.

In that sense, the demographic-epidemiological transition, characterized by high prevalence of diseases and risk factors related to lifestyle, has demanded a profound transformation of the health care model. Increased service provision and prevention and health promotion actions are needed, based on scientific evidence, covering innovative information, education and communication initiatives ${ }^{(1)}$.

This situation reveals the need for flexibility to ensure adaptation to new changes in a rapidly changing world, new media and knowledge production strategies and technologies for comprehensive care delivery to the population. This requires the development of a new field of knowledge, entitled Health Technology Assessment (HTA).

HTA includes studies on drugs, equipment and technical procedures, organizational, educational, information and support systems, as well as care programs and protocols, through which health care and actions are offered to the population.

In the global context, HTA emerged in the 1960's as a tool used for decision making by health professionals and other disciplines. In Brazil, this movement occurred in the 1980's, and educational and research institutions were pioneers in the development of HTA research.

Nursing, as a discipline attentive to the needs of a changing world and recognizing that the professionalcentered, individualized and real-time care model will not be able to provide care effectively and efficiently, has made efforts in human resources training with a view to proper information and communication technology use and research development with new cognitive designs.

These studies suggest that new technologies, such as concept maps used to teach people with CNCDs and undergraduate students, multimedia resources for teaching specific procedures, educational games in disease prevention, hypertext and simulators for training and evaluation of faculty and students, weblogs and virtual communities like Orkut, as teaching tools, SMS and use of phones as an educational tool for people with CNCDs, development of protocols to guide clinical practice, forms of humanized care, innovative playful strategies for strengthening self-care actions, among others, have changed the concepts and resources used in the teachinglearning process. These tools permit the dissemination of scientific evidence-based knowledge to a significant number of citizens, at a reasonable cost and, at the same time, breaking the space-time barrier. 
In Brazil, since 2003, the Ministry of Health, through the Council for Science, Technology and Innovation in Health, established a Permanent Work Group that aims to promote and disseminate priority research in HTA, train managers, create an international cooperation network for HTA knowledge production and strengthened support for decision-making on health technologies ${ }^{(2-3)}$.

Thus, health and health-related professionals need to reflect on knowledge production and technology use, considering the prevalence of CNCDs in the population, as well as aspects related to its operational cost and the monitoring of the load and trend of these diseases.

It is thus recognized that strategies are needed to strengthen scientific and technological production through collaborative networks, partnerships with other universities and establishment of several interdisciplinary centers with a view to research development with new cognitive designs in care delivery to people with CNCDs. Nursing should embrace the challenge of increasing scientific production in health technology and providing new tools for information and communication in care to Chronic Non-Communicable Diseases, in order to increase the efficacy of health services.

\section{References}

1. Buss PM. Promoção e educação em saúde no âmbito da Escola de Governo em Saúde. Cad. Saúde Pública 1999;15(Suppl 2):177-85.

2. Ministério da Saúde, Secretaria de Ciência e Tecnologia e Insumos Estratégicos, Departamento de Ciência e Tecnologia. Avaliação de Tecnologias em Saúde: Institucionalização das ações no Ministério da Saúde. Rev Saúde Pública 2006;40(4):743-7.

3. Lino MM, Backes VMS, Canever BP, Ferraz F, Prado ML. Profile of scientific and technological production in nursing education research groups in the south of Brazil. Rev. Latino-Am. Enfermagem 2010;18(3):452-8.

Maria Lúcia Zanetti is member of the Editorial Board of the Latin American Journal of Nursing, and Associate Professor of the University of São Paulo at Ribeirão Preto College of Nursing, WHO Collaborating Centre for Nursing Research Development, Brazil, e-mail: zanetti@eerp.usp.br. 